\title{
AN AUTOMATIC APPROACH FOR SELECTING NEW Ho EMISSION-LINE GALAXIES
}

\author{
O. ALONSO, J. ZAMORANO, M. REGO, J. GALLEGO and A.G. VITORES \\ Dpto. de Astrofisica \\ Universidad Complutense de Madrid \\ 28040 Madrid \\ Spain
}

\section{Introduction}

The most unambiguous way to discover new emission-line galaxies (ELGs) is directly by the presence of their lines, using objective-prism plates of adequate resolution. The first survey using this technique was developed by Smith in 1975 with the $0.6 \mathrm{~m}$ CTIO Curtis Schmidt Telescope. The Universidad Complutense de Madrid (UCM) is carrying out a survey of ELGs with the Schmidt Telescope at Calar Alto (Almeria, Spain) using the presence of $\mathrm{H} \alpha$ in emission in IIIa-F prism plates as selection criterion. The observational procedure and results are described in Rego et al. 1989; Zamorano et al. 1990; Zamorano et al. 1993.

The standard procedure is to scan the plates by eye, with a low-power binocular microscope. The use of different subjective criteria in the visual inspection can produce biased samples. Moreover, the visual scan does not produce any quantitative measure but only a sample of candidates. Therefore, in order to obtain an observer-independent sample and to extract the largest amount of information from the original plates, 2 plates have been scanned with the MAMA machine ${ }^{\dagger}$ (Guibert \& Moreau 1991; Moreau 1992) with the aim of developing an automatic procedure to detect ELGs spectra.

\section{Plate Material, Digitization and Data Reduction}

In this work, we have used two new plates from the UCM survey. Direct and prism plates were taken with the $80 / 120 \mathrm{~cm} \mathrm{f} / 3$ Schmidt Telescope of the Calar Alto German-Spanish Observatory in Almeria, Spain (Birkle 1984). The IIIa-F emulsion and RG630 filter select a useful spectral range from 6400 to $6850 \AA$. The use of a $4^{\circ}$ objective-prism provides a dispersion of $1950 \AA / \mathrm{mm}$ at $\mathrm{H} \alpha$. This instrumental setup is able to record the $\mathrm{H} \alpha$ line in emission for objects up to $\mathrm{z}=0.04$.

These plates were scanned with the MAMA machine in December 1992 using a pixel size and

Based on observations taken at Calar Alto Observatory (Almería, Spain)

' MAMA (Machine Automatique à Mesurer pour l'Astronomie) is developed and operated by CNRS/INSU (Institut National des Science de l'Univers) and located at Observatoire de Paris. 
sampling step of $10 \mu \mathrm{m}$. Object catalogues with position, density flux, area and shape parameters for all the images on the plates, including emulsion defects, were created by MAMA. In addition, the prism plate was digitized and fully stored in $22 \times 221024 \times 1024$ pixel FITS images, implying more than 1 Gbyte of raw data. Mosaic images were created extracting $61 \times 21$ pix. boxes around each object on the original FITS images. This allows a fast and easier access to the spectra, and produces a high reduction of the space for the data storage. All the useful information can be saved in 50 Mbyte, $5 \%$ of the initial volume of data.

The unidimensional spectra were extracted using marginal sums perpendicular to the direction of the dispersion. Only the 5 central scans of each spectrum were used. For all the spectra, the red emulsion cut-off was positioned at the same value using the algorithm given by Borra et al. (1987). The original prism spectra were smoothed, filtering the high frequencies in the Fourier space. We have used the Fourier filter because it doesn't modify the weak emission-lines of the original spectra, as box or median filters do.

The presence of the $\mathrm{H} \alpha+[\mathrm{NII}]$ blend in emission is used as a criterion to select ELGs spectra. As we show in Fig. 1, the stellar spectra can be modelled by a continuum. However, the continuum determination for the ELGs spectra reveal the emission-line. The spectral range together with the prism dispersion produce very short spectra (30 pixels approx.), and the continuum determination is a difficult task because of the high number of spectral shapes. The continua of the spectra can be determined, in the majority of the cases, using spline curves. The use of an iterative algorithm to reject the points with large deviations provides good fits including spectra with strong lines in emission (top of Fig. 1).

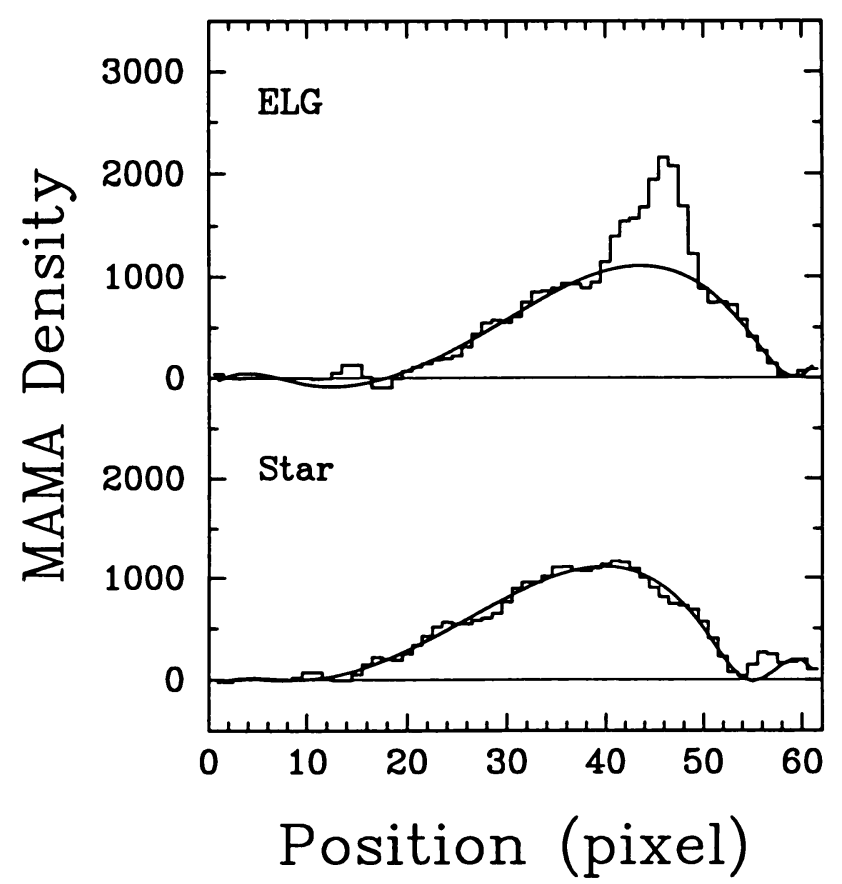

Figure 1. Typical stellar and emission-line galaxy (ELG) prism spectra together with the calculated continua. The $\mathrm{H} \alpha+[\mathrm{NII}]$ blend in emission can be seen clearly over the estimated continuum in the galaxy spectrum. 


\section{Results}

The objects with largest residua between the spectrum and the calculated continuum were selected and tested in order to reject the wrong selections due to either incorrect continuum determination or emulsion defects. The objects finally selected as ELGs were visually examined on the prism plate. After the visual check, 9 out of 10 candidates were confirmed. The spectrum rejected showed a clear line in the digitized image, but not in the prism plate, probably produced by a blemish at the moment of the scan.

Moderate spectroscopic observations of two candidates, which are not included in the Catalogue of Principal Galaxies (Paturel et al. 1989), have been obtained with the $2.2 \mathrm{~m}$ telescope at Calar Alto Observatory in August 1993. Figures 2 and 3 show the prism and slit spectra. UCM1506+2821 is a bright object with a strong line in the prism spectrum. The slit spectrum presents a high reddening and shows the characteristics of a Starburst Nucleus. The second object, UCM1458+2820, is fainter in the red region, although it shows an important excess in the blue. In this case, the spectrum is typical of an HII galaxy. The broad emission line in the prism spectrum is due to the extension of the emitting region.

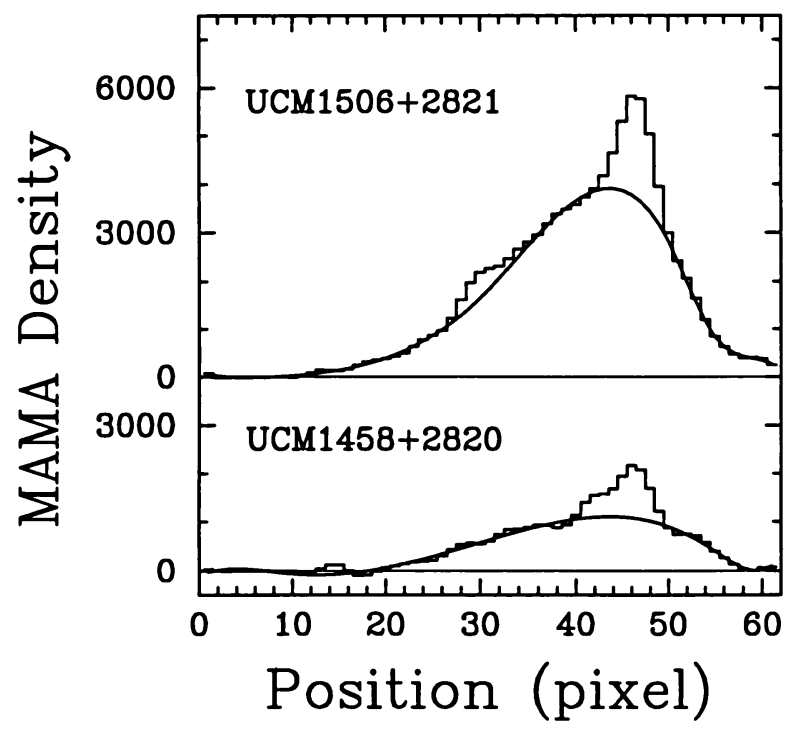

Figure 2. Prism spectra of the 2 new UCM galaxies automatically identified. The spectral range covers from 6400 to $6850 \AA$.

\section{Conclusion}

We have developed a fully automatic algorithm to identify ELGs spectra from objective-prism plates. The continuum of the spectra has been derived fitting spline curves by using an iterative process. The spectra are selected by the presence of large differences compared with the calculated continuum, and all of them present clear emission-line features, although some cases are due to emulsion defects or incorrect continuum determination. The confirmation of 2 of the candidates with spectroscopic observations as new ELGs validates the success of the procedure. 


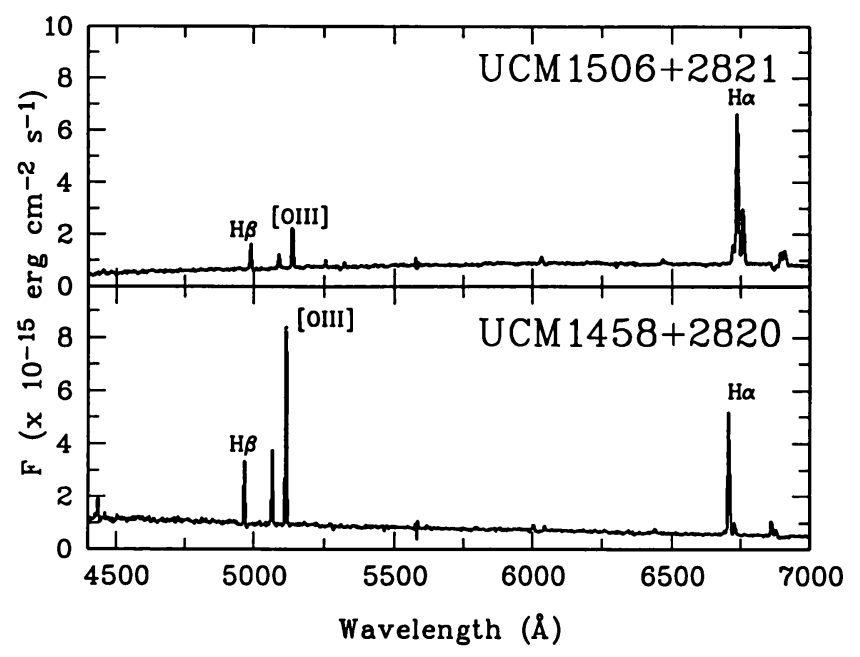

Figure 3. Slit spectra of the 2 new UCM galaxies.

\section{References}

Birkle, K., 1984. In 'Astronomy with Schmidt-Type Telescopes', ed. M. Capaccioli, IAU coll. 78, p. 203.

Borra, E.F., Edwards, G., Petrucci, F., Beauchemin, M., Brousseau, D., Grondin, L. and Beaulieu, A., 1987. Publ. Astron. Soc. Pacific, 99, 535.

Guibert, J. and Moreau, O., 1991. The Messenger, 64, 69.

Moreau, O., 1992. PhD Thesis, Université de Paris 7.

Paturel, G., Fouque, P., Bottinelli, L. Gouguenheim, L., 1989. Astron. Astrophys. Suppl., 80, 299.

Rego, M., Zamorano, J and Gonzalez-Riestra, R., 1989. Astron. Astrophys. Suppl., 79, 443.

Smith, M.G., 1975. Astrophys. J., 202, 591.

Zamorano, J., Rego, M., Gonzalez-Riestra, R. and Rodriguez, G., 1990. Astrophys. Space Sci., 170, 353.

Zamorano, J., Rego, M., Gallego, J., Vitores, A.G., Gonzalez-Riestra, R. and Rodríguez-Caderot, G., 1993. Astrophys. J. Suppl., submitted. 